\title{
Ionic liquid modified graphene nanoplatelets electrode for high performance supercapacitor
}

\author{
Mohammad Yasir, S. A. Hashmi* and Manoj K. Singh \\ Department of Physics \& Astrophysics, University of Delhi, Delhi-110007, India \\ *Email: sahashmi@physics.du.ac.in
}

Now a days, supercapacitors have been drawn global attention due to its high power delivering ability and excellent cyclic performance [1,2]. The electrical double layer capacitors (EDLCs), a special class of supercapacitor employs carbonaceous materials (e.g. activated carbon, carbon nanofibers, and CNTs etc.) as porous electrodes with large specific surface area [1]. Recently, Graphene has emerged as one of the most promising alternative electrode material in device application such as batteries, fuel-cells and supercapacitors [3].

In the present study, we report the fabrication and characterization of supercapacitor based on ionic liquid modified GNP electrodes and gel polymer electrolyte. The gel polymer electrolyte was prepared using solution-cast method with ionic liquid 1-butyl-3methylimidazolium bis (trifluoromethylsulfonyl) imide (BMITFSI) immobilized in poly(vinylidene fluoride-cohexafluoropylene) (PVdF-HFP).
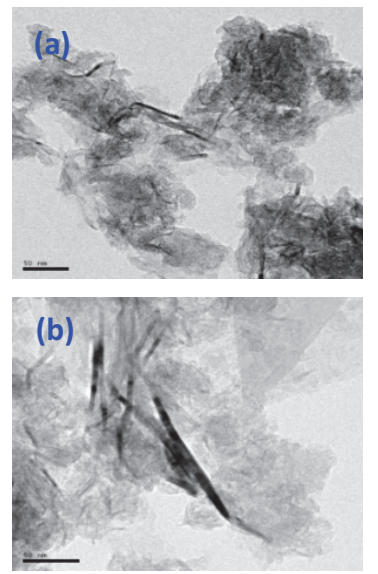

Figure 1: TEM images both at $50 \mathrm{~nm}$ scale of (a) GNP and (b) modified GNP electrode material.

The gel electrolyte was characterized using electrochemical method namely a.c. Impedance spectroscopy and Linear sweep voltammetry (LSV) for evaluating the ionic conductivity and electrochemical stability window respectively.
The performances of supercapacitor were characterized using electrochemical impedance spectroscopy, cyclic voltammetry and galvanostatic charge-discharge techniques.

Figure 1 shows the TEM images of GNP and modified GNP electrode material at scale 50 $\mathrm{nm}$. The impedance and CV pattern of the typical EDLC cell with ionic liquid modified GNP electrode and gel polymer electrolyte are shown in Figure 2 (a) \& (b) respectively. The steep rise behavior shows the capacitive nature of the system. The capacitance value has been achieved to $\sim 70.4 \mathrm{~F} \mathrm{~g} \mathrm{~g}^{-1}$ with modified GNP electrode (Figure 2 a). The CV pattern shows almost rectangular shapes for the scan rates up to $1000 \mathrm{mV} \mathrm{s}^{-1}$ in Figure 2(b) which shows the high rate capability of the supercapacitor.
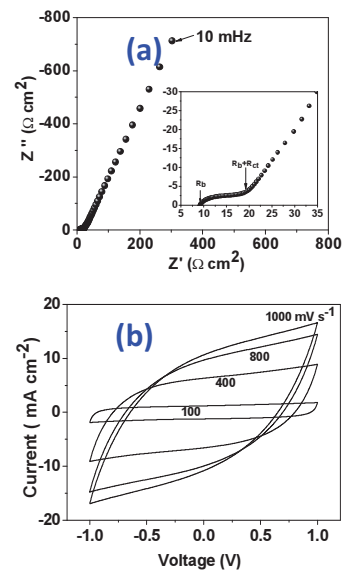

Figure 2: (a) Typical impedance and (b) cyclic voltammetry plot of EDLC recorded at room temperature

Keywords: Ionic liquid, gel polymer electrolyte, modified GNP.

\section{References}

1. F. Beguin, and E Frackowiak; Supercapacitor: materials, system, applications. Singapore: WileyVCH Verlag GmbH \& Co. KGaA; 2013.

2. M. K. Singh, M. Suleman, Y. Kumar, and S. A. Hashmi; Energy 80(2015)465.

3. M. Pumera; Energy Environ Sci 4(2011)668 